Modeling 3D PCMI using the Extended Finite Element Method with higher order elements

B.W. Spencer, INL

W. Jiang, INL

March 31, 2017

Approved for Public Release
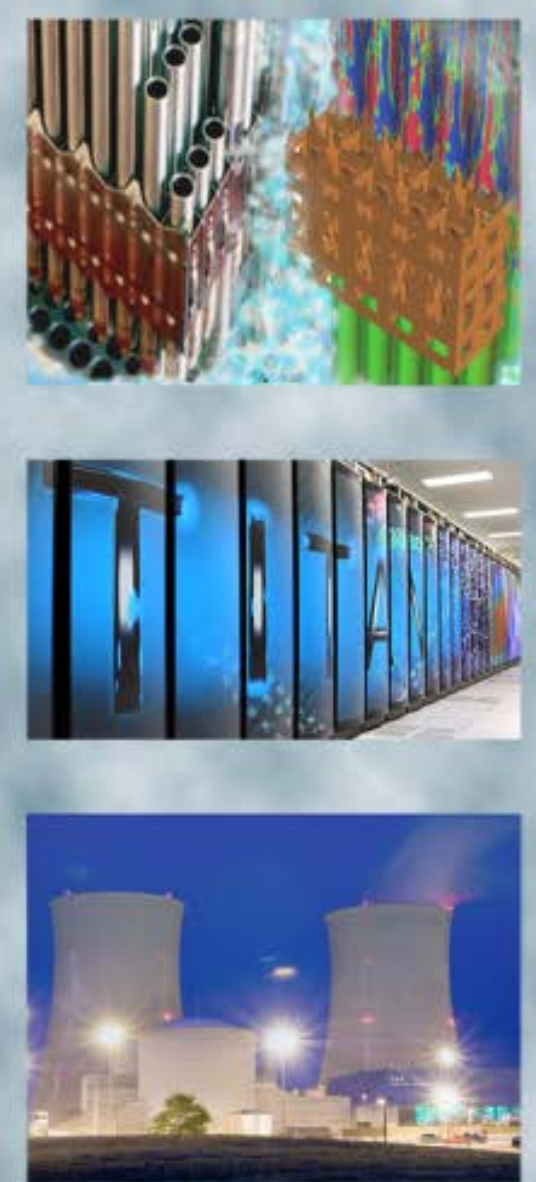
Please complete sections appropriate for this record.

REVISION LOG

\begin{tabular}{|c|c|c|l|}
\hline Revision & Date & Affected Pages & \multicolumn{1}{c|}{ Revision Description } \\
\hline 0 & $03 / 31 / 2017$ & All & Initial Release \\
\hline & & & \\
\hline & & & \\
\hline & & & \\
\hline
\end{tabular}

\section{Document pages that are:}

Export Controlled ___ None

IP/Proprietary/NDA Controlled_____ None

Sensitive Controlled__ None

Unlimited: ALL

\section{Requested Distribution:}

To:

Copy:

This report was prepared as an account of work sponsored by an agency of the United States Government. Neither the United States Government nor any agency thereof, nor any of their employees, makes any warranty, express or implied, or assumes any legal liability or responsibility for the accuracy, completeness, or usefulness of any information, apparatus, product, or process disclosed, or represents that its use would not infringe privately owned rights. Reference herein to any specific commercial product, process, or service by trade name, trademark, manufacturer, or otherwise, does not necessarily constitute or imply its endorsement, recommendation, or favoring by the United States Government or any agency thereof. The views and opinions of authors expressed herein do not necessarily state or reflect those of the United States Government or any agency thereof. 


\title{
Modeling 3D PCMI using the Extended Finite Element Method with higher order elements
}

\author{
CASL FY17 Letter Report \\ INL/MIS-17-41609
}

\author{
B. W. Spencer, W. Jiang \\ Fuels Modeling and Simulation \\ Idaho National Laboratory \\ P.O. Box 1625 \\ Idaho Falls, ID 83415-3840
}

March 31, 2017

\section{Introduction}

The eXtended Finite Element Method (XFEM) [1, 2] is a powerful method to represent arbitrary propagating discrete cracks in finite element models without remeshing. Work has been underway to implement XFEM in the MOOSE framework and enable its application to fuel fracture problems within BISON.

XFEM was initially applied to model fracture propagation in ceramic nuclear fuel, and was used in models that contained no cladding. Under CASL funding in FY16, the implementation of the XFEM in BISON was expanded to permit its use for the analysis of cladding stresses in 2D models of the region in the vicinity of a crack in the fuel adjacent to the cladding[3,4] due to pellet-cladding mechanical interactions (PCMI). The results from this work are very encouraging, as the effects of discrete cracks are captured reasonably well, especially with mesh refinement, and these cracks can be defined independently of the finite element mesh.

The initial work modeling PCMI used linear finite elements, and was limited to 2D applications. XFEM has the potential to be particularly useful in 3D PCMI analyses, where generating meshes conforming to cracks is more difficult. There are two goals of the work documented here: to improve the accuracy of the contact solulutions with less need for mesh refinement when using the XFEM for PCMI analysis, and to extend that capability to 3D PCMI models.

For all BISON simulations where there are interactions between curved surfaces of the fuel and cladding, quadratic elements are preferred over linear elements due to the fact that they are more accurate to represent the curved geometry and often leads to much better convergence. These curved surfaces are typically encountered when simulating light water reactor (LWR) fuel when the fuel/cladding system is either represented using a 2D cross-section model or a 3D model.

The initial implementation of XFEM only worked for linear elements. For the present effort, it was determined that to accomplish the goal of improving the fidelity of contact results both adjacent to cut elements as well as away from cut elements, the most impactful development would be to enable the use of higher-order elements with XFEM.

This report documents the recent development to enable XFEM to work with higher order elements. It also demonstrates the application of higher order (quadratic) elements to both 2D and 3D models of PCMI problems, where discrete fractures in the fuel are represented using XFEM. The modeling results demonstrate the ability of the higher

order XFEM to accurately capture the effects of a crack on the response in the vicinity of the intersecting surfaces of cracked fuel and cladding, as well as represent smooth responses in the regions away from the crack. 


\section{Higher order elements for XFEM}

As a widely adopted technique to introduce Heaviside discontinuities, the phantom node method was used to develop an implementation of the XFEM in the MOOSE framework. In the phantom node method, elements intersected by a crack are deleted and replaced by two elements that occupy the same physical locations as the original elements. A portion of the domain of each of these new elements (partial elements) represents physical material, while the remainder does not. The combined physical portions of the partial elements cover the entire domain of the original element that was split. The nodes that are connected to the non-physical portions of those elements are known as phantom nodes. The initial implementation of the mesh cutting algorithm did not account for the edge nodes and interior nodes in higher order elements. As a result, before the present work, only linear elements, such as 4-node quadrilateral element and 8-node hexahedral element, could be used in MOOSE with XFEM.

To allow the use of higher order elements in conjunction with XFEM, the procedure for creating child elements and duplicating the nodes have been redesigned to accommodate those higher order nodes. An example of the overlapping element formulation for a cut 9-node quadrilateral element is shown in Figure 1. In the process of creating child elements, the center point and the normal of the cut line is identified. Based on the cut line location, the nodes which lie within the physical domain are categorized as physical nodes and the remaining ones as phantom nodes. With this modified algorithm, XFEM has been developed to work with quadratic elements for both 2D and 3D, including 8-node quadrilateral element, 6-node triangular element, 10-node tetrahedral element, 20-node hexahedral element and 27 node hexahedral elements.

The finite element discretization is now constructed on this overlapping domain. The approximation and the weighting functions are then given by:

$$
u^{h}=\sum_{\mathrm{m}} \sum_{i \in I^{\mathrm{m}}} H^{\mathrm{m}} N_{i} u_{i} \quad w^{h}=\sum_{\mathrm{m}} \sum_{i \in I^{\mathrm{m}}} H^{\mathrm{m}} N_{i} w_{i}
$$

where $I^{\mathrm{m}}$ is the set of all nodes whose support overlap the domain $\Omega^{\mathrm{m}}$ and $H^{\mathrm{m}}$ is the characteristic function given by

$$
H^{\mathrm{m}}(\mathbf{x})= \begin{cases}1 & \text { if } \mathbf{x} \in \Omega^{\mathrm{m}} \\ 0 & \text { otherwise }\end{cases}
$$

The solution field is interpolated on the partial elements using the standard shape functions. The integration is weighted by the volume fraction of the physical domain. The main challenge in the phantom node method is developing an algorithm to correctly connect the partial elements split by a crack. Neighboring elements that share a common cracked edge should share physical and phantom nodes. For higher order elements, those higher order nodes have to be taken into account in order to successfully reconnect the partial elements.
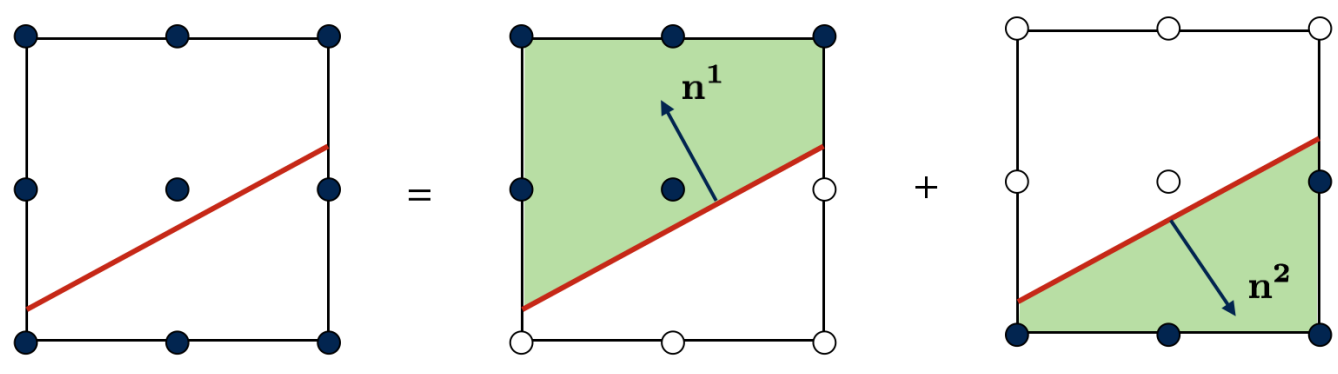

Figure 1: Overlapping element formulation for a 9-node quadrilateral element cut by a crack. The black circles are the physical nodes and the hollow circles are the phantom nodes.

\section{Applications of XFEM with higher-order elements to PCMI Modeling}

To demonstrate the application of quadratic elements, two examples are shown here: one to a 2D LWR fuel problem, and one to an equivalent 3D model of the same problem. Both of these are local models intended to capture local effects 
due to the presence of a crack in the fuel. These models rely on another global 2D model of the full fuel rod to supply time histories of plenum gas properties, using the procedure described in [5]. These models are subjected to the same transient power history described in that paper.

\section{D Example}

To demonstrate the relative improvements of using quadratic elements over linear elements in a 2D cross-section model, a quarter-symmetry model of a BWR fuel pellet and caldding has been run using both linear and quadratic elements. In the quadratic element model shown here, 9-noded elmements were used, but either 8- or 9-noded elements can be used. These models employ independent generalized plane strain constraints on the fuel and cladding. These models both have meshes with identical element discretizations, and which were constucted to be quite coarse to highlight the improvements due to the use of quadratic elements. The only difference between the meshes is that the 9noded element mesh has additional mid-edge and mid-face nodes, which are located on the curved boundaries for those elements that are located on boundaries. These meshes were also intentionally constructed so that the discretizations for fuel and cladding that do not have coincident nodes to show how quadratic elements adequately capture the interactions of the curved surfaces in these cases, while linear elements do not.

Figure 2 shows a comparison of the temperature solutions at the end of the irradiation history for these two models. It is important to note that the Paraview code used to visualize the results represents higher-order elements by subdividing them after the filter to show XFEM cuts is applied, so the quadratic element mesh appears to be more refined than the linear element mesh, even though the two meshes are identical, with the exception of the additional mid-edge and mid-face nodes in the quadratic mesh. These results clearly show the uneven gaps between the fuel and cladding in the linear case, which are almost completely removed in the case with the quadratic elements. These gaps can have a significant effect on the thermal solution because of the importance of the gap size on its conductance. Some noise is seen in the fuel temperature fields on the outer rim of the fuel, and this noise is reduced in the quadratic element case.

Local irregularities in the solution fields with linear meshes around the contact surfaces are especially evident when the contact pressure field is plotted. Figure 3 shows a comparison of the contact pressure field for the linear and quadratic meshes. Although there are still some minor irregularities in the contact pressure in the quadratic mesh case, they are significantly smaller than the irregularities for the linear mesh.
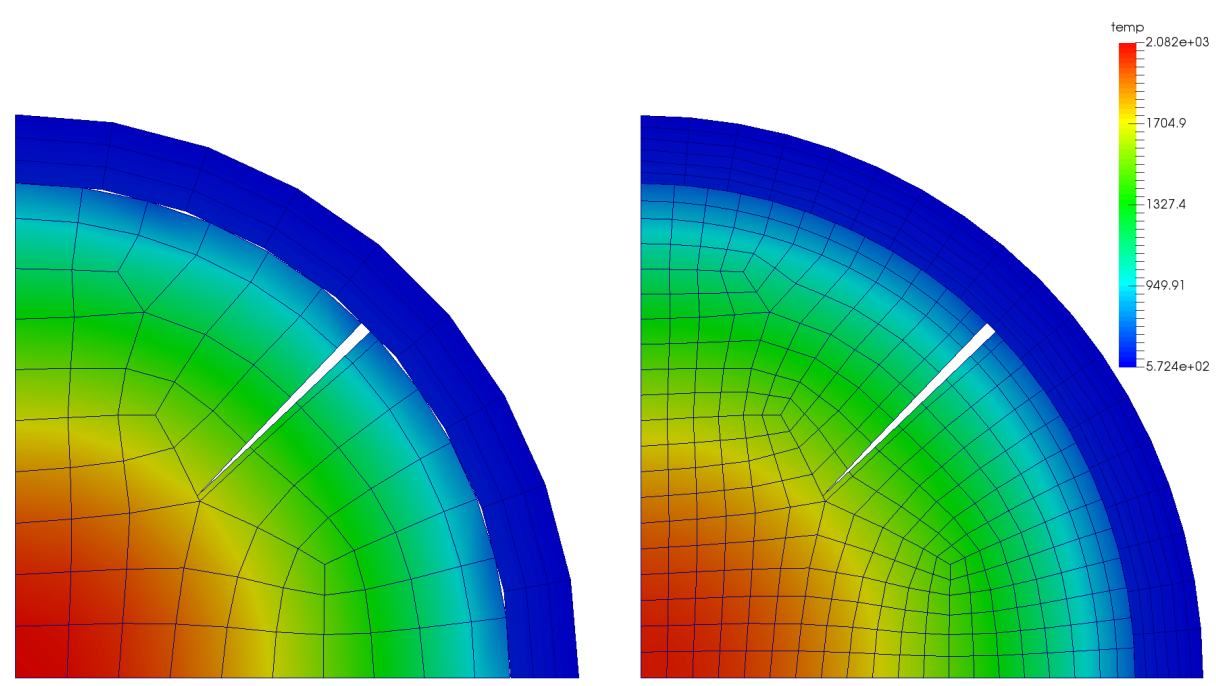

Figure 2: Comparison of temperatures (K) calculated with XFEM using 4-noded linear elements (left) and 9-noded quadratic elements (right). The same mesh is used in both cases (although the quadratic element mesh appears more refined due to visualization artifacts). 

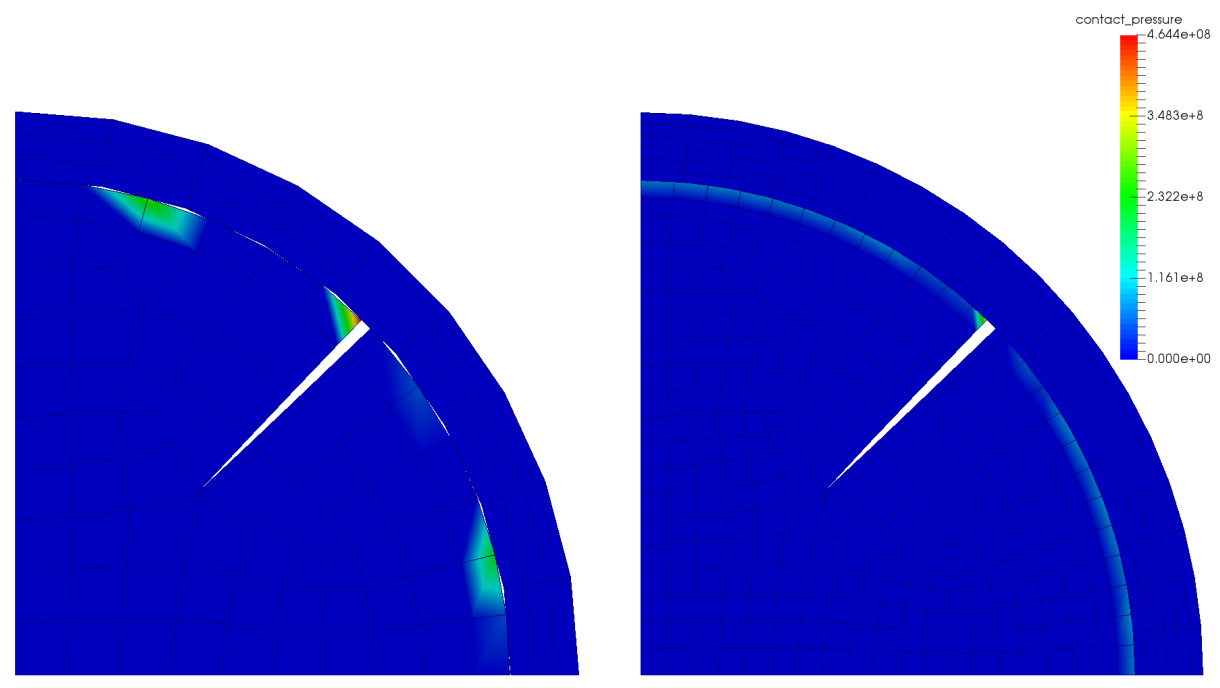

Figure 3: Comparison of contact pressures (Pa) calculated with XFEM using 4-noded linear elements (left) and 9noded quadratic elements (right). The same mesh is used in both cases. The same mesh is used in both cases (although the quadratic element mesh appears more refined due to visualization artifacts).

\section{D Example}

A simplified model of a single pellet and cladding equivalent to the $2 \mathrm{D}$ case was developed to demonstrate the use of XFEM for PCMI analysis in 3D. As for the 2D case, this model uses quarter symmetry, and models a single radial crack. The 3D model represents the pellet using an additional symmetry plane through the center of the pellet in the axial direction. 27-noded hexahedral elements were used in this model, which was subjected to the same loading history as the 2D model.

Figure 4 shows a contour plot of the temperature on the fuel and cladding, and Figure 5 shows contours of the von Mises stress in the cladding as observed from the interior and exterior at a point in time mid-way through the transient history. Although the mesh is fairly coarse in this model, it captures local stress concentrations in the cladding exterior at the top of the pellet adjacent to the crack, as would be expected.

\section{Conclusion}

Improvements have been made to the mesh cutting algorithm employed for XFEM in MOOSE and BISON to permit the use of higher order elements. This significantly imrpoves the solution both in the vicinity of fuel cracks as well as in other areas of the fuel/cladding interface away from the fuel. This has been demonstrated on both 2D and 3D models of PCMI.

XFEM will be particulary useful in 3D PCMI simulations, where including the geometries of discrete cracks in the 3D mesh can be challenging. Because XFEM permits the representation of cracks independent of mesh geometry, there is no need to construct meshes that conform to a given crack geometry.

These analyses were performed on relatively simple, coarse models to demonstrate the utility of this technique. Future work will focus on the application of this technique to larger, more realistic models to study the effects of general crack geometries on PCMI-induced stresses. 


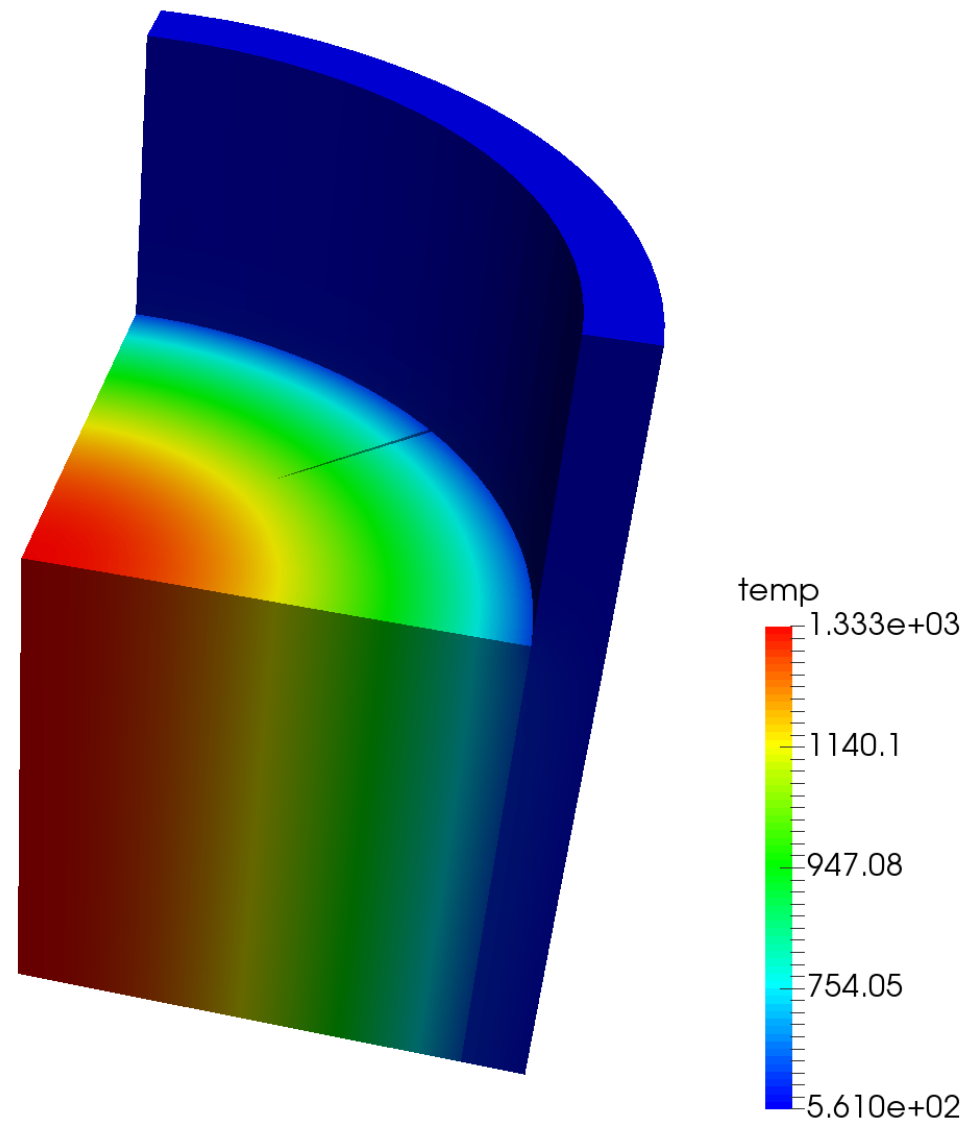

Figure 4: 3D model, showing contours of the temperature $(\mathrm{K})$ in the fuel and cladding 

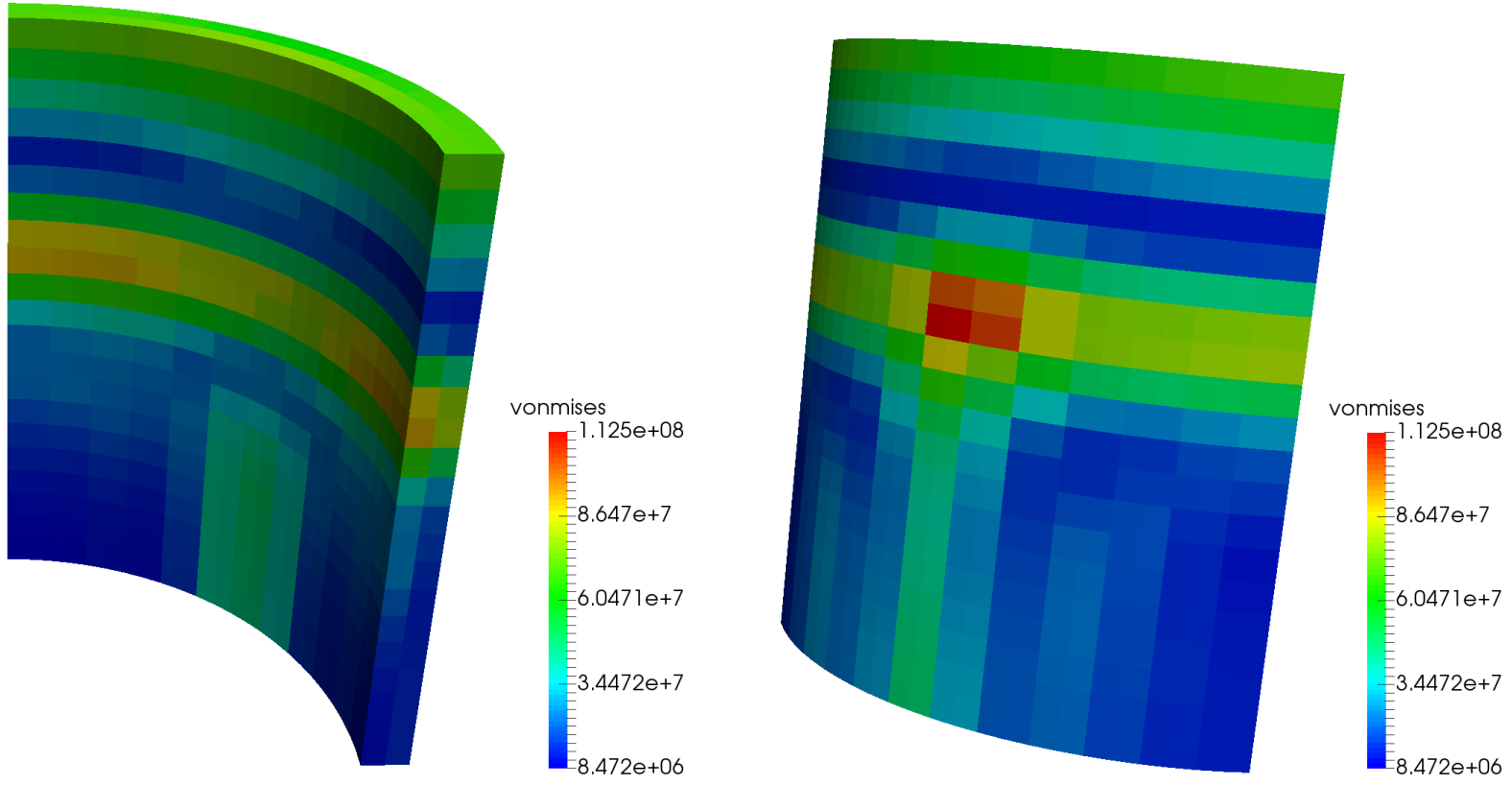

Figure 5: Contour plots of the von Mises stress $(\mathrm{Pa})$ in the cladding as seen from the inside (left) and outside (right)

\section{References}

[1] T. Belytschko and T. Black. Elastic crack growth in finite elements with minimal remeshing. International Journal for Numerical Methods in Engineering, 45(5):601-620, June 1999.

[2] Nicolas Moës, John Dolbow, and Ted Belytschko. A finite element method for crack growth without remeshing. International Journal for Numerical Methods in Engineering, 46(1):131-150, September 1999.

[3] Benjamin W. Spencer, Wen Jiang, John E. Dolbow, and Christian Peco. Modeling PCMI and Interactions Across Interfaces Modeled using the Extended Finite Element Method CASL FY16 Letter Report. Technical report, Idaho National Laboratory, July 2016.

[4] Benjamin W. Spencer, Wen Jiang, John E. Dolbow, and Christian Peco. Pellet cladding mechanical interaction modeling using the extended finite element method. In Proceedings of Top Fuel 2016, Boise, ID, September 2016.

[5] B.W. Spencer, R.L. Williamson, D.S. Stafford, S.R. Novascone, J.D. Hales, and G. Pastore. 3D modeling of missing pellet surface defects in BWR fuel. Nuclear Engineering and Design, 307:155-171, October 2016. 